\title{
A DISCONJUGACY CRITERION FOR LINEAR SCALAR DIFFERENTIAL OPERATORS
}

\author{
JAMES S. MULDOWNEY ${ }^{1}$
}

\begin{abstract}
It is shown that if a linear scalar differential operator is not disconjugate on an interval then each member of a certain family of first order vector differential equations has an oscillatory solution. Thus any condition which guarantees the nonoscillation of a member of the family is a disconjugacy criterion for the scalar operator. The form of the vector systems is convenient for the use of nonoscillation conditions developed by Nehari, Schwarz and Friedland.
\end{abstract}

Let $L$ denote the $n$th order linear differential operator

$$
L f=f^{(n)}+p_{1}(t) f^{(n-1)}+\cdots+p_{n}(t) f
$$

where the coefficients $p_{i}$ are continuous real valued functions on a real interval $I$. The operator $L$ is said to be disconjugate on $[a, b] \subset I$ if the only solution of $L f=0$ having $n$ zeros or more (counting multiplicities) in $[a, b]$ is the zero solution. Consider also a general first order linear system of differential equations

$$
x^{\prime}=A(t) x
$$

where $A(t)$ is an $n \times n$ matrix of real valued continuous functions on $I$. A nontrivial vector solution $x(t)$ of $(2)$ is called oscillatory on $[a, b]$ if each component of $x(t)$ has a zero in $[a, b]$. The system (2) is nonoscillatory if it has no oscillatory solution.

The following lemma shows that if $L$ is not disconjugate on $[a, b]$ then certain systems (2) have oscillatory solutions. It is assumed that $u(t)=$ $\operatorname{col}\left(u_{1}(t), \cdots, u_{n}(t)\right)$ is any $C^{n}$ vector valued function on $[a, b]$ such that

(3) $W\left(u_{1}, \cdots, u_{n}\right) \neq 0, \quad W\left(u_{1}, \cdots, \hat{u}_{k}, \cdots, u_{n}\right) \neq 0, \quad k=1, \cdots, n$, where $W\left(\phi_{1}, \cdots, \phi_{m}\right)$ denotes the wronskian determinant $\operatorname{det}\left\{\phi_{i}^{(j-1)}\right\}, i, j=$ $1, \cdots, m$, and $\left(u_{1}, \cdots, \hat{u}_{k}, \cdots, u_{n}\right)=\left(u_{1}, \cdots, u_{k-1}, u_{k+1}, \cdots, u_{n}\right)$; also $v(t)=\operatorname{col}\left(v_{1}(t), \cdots, v_{n}(t)\right)$ where

$$
v_{k}=W\left(u_{1}, \cdots, \hat{u}_{k}, \cdots, u_{n}\right) / W\left(u_{1}, \cdots, u_{n}\right)
$$

and the operators (or order $n-1) U_{k}, k=1, \cdots, n$, are defined by

$$
U_{k} f=W\left(u_{1}, \cdots, \hat{u}_{k}, \cdots, u_{n}, f\right) / W\left(u_{1}, \cdots, \hat{u}_{k}, \cdots, u_{n}\right) .
$$

Received by the editors August 2, 1976.

AMS (MOS) subject classifications (1970). Primary 34C10.

Key words and phrases. Disconjugacy, nonoscillation.

${ }^{1}$ Research supported by NRC Grant A-7197. 
LemMa. Suppose that $\left(u_{1}(t), \cdots, u_{n}(t)\right)$ are any $C^{n}$ functions satisfying (3) on $[a, b]$ and that each of the operators $U_{k}$ is disconjugate on $[a, b]$. Then if $L$ is not disconjugate on $[a, b]$ the system

$$
x_{j}^{\prime}=v_{j} \sum_{k=1}^{n}(-1)^{n-k-1}\left(L u_{k}\right) x_{k}, \quad j=1, \cdots, n,
$$

has an oscillatory solution on $[a, b]$.

Proof. The following wronskian identities are required:

$$
\begin{aligned}
& L f=\frac{W\left(u_{1}, \cdots, u_{n}, f\right)+\sum_{k=1}^{n} W\left(u_{1}, \cdots, \hat{u}_{k}, \cdots, u_{n}, f\right)(-1)^{n-k} L u_{k}}{W\left(u_{1}, \cdots, u_{n}\right)}, \\
& \begin{aligned}
\frac{W\left(u_{1}, \cdots, u_{n}, f\right)}{W\left(\dot{u}_{1}, \cdots, u_{n}\right)} \\
\quad=\left[\frac{W\left(u_{1}, \cdots, \hat{u}_{k}, \cdots, u_{n}, f\right)}{W\left(u_{1}, \cdots, u_{n}\right)}\right]^{\prime} / \frac{W\left(u_{1}, \cdots, \hat{u}_{k}, \cdots, u_{n}\right)}{W\left(u_{1}, \cdots, u_{n}\right)} .
\end{aligned}
\end{aligned}
$$

Both identities follow from the observation that if $L$ and $M$ are two linear differential operators of order $n$ with leading coefficients 1 (as in (1)) then $L=M$ if $L \phi_{i}=M \phi_{i}, i=1, \cdots, n$, for some (and hence for every) system of functions $\left(\phi_{1}, \cdots, \phi_{n}\right)$ such that $W\left(\phi_{1}, \cdots, \phi_{n}\right) \neq 0$. From (7) and (8) it follows that if

$$
x_{k}=W\left(u_{1}, \cdots, \hat{u}_{k}, \cdots, u_{n}, f\right) / W\left(u_{1}, \cdots, u_{n}\right)=v_{k} U_{k} f
$$

then

$$
L f=\frac{x_{j}^{\prime}}{v_{j}}+\sum_{k=1}^{n}(-1)^{n-k}\left(L u_{k}\right) x_{k}, \quad j=1, \cdots, n,
$$

and so, if $L f=0,\left(x_{1}, \cdots, x_{n}\right)$ satisfies (6). If, in addition, $f$ is not identically zero, then not all of the $x_{k}$ 's are identically zero, since in that case $W\left(u_{1}, \cdots, u_{n}, f\right) / W\left(u_{1}, \cdots, u_{n}\right)=0$ from (7) and, thus, $f=c_{1} u_{1}$ $+\cdots+c_{n} u_{n}$; but

$$
W\left(u_{1}, \cdots, \hat{u}_{k}, \cdots, u_{n}, f\right) / W\left(u_{1}, \cdots, u_{n}\right)=x_{k}=0
$$

implies $c_{k}=0, k=1, \cdots, n$, contradicting the assumption that $f$ is not identically zero. Finally, if $f$ has $n$ zeros or more in $[a, b]$, then by the Pólya mean value theorem (cf. [8]) each of the functions $x_{k}$ has a zero in $[a, b]$ since $x_{k}=v_{k} U_{k} f$, and $U_{k}$ is disconjugate on $[a, b]$. Thus $\operatorname{col}\left(x_{1}, \cdots, x_{n}\right)$ is an oscillatory solution of (6).

The lemma shows that, subject to the restrictions on $u$, any nonoscillation condition for (6) is a disconjugacy criterion for $L$. In a series of papers Nehari, Schwarz and Friedland (e.g., cf. [6], [7], [9], [3]) best possible numbers $c_{r p}(n)$ are found such that 


$$
\int_{a}^{b}\|A\|_{r p}<c_{r p}(n)
$$

implies (2) is nonoscillatory on $[a, b]$, where

$$
\begin{aligned}
& \|A\|_{r}=\sup \frac{\|A x\|_{p}}{\|x\|_{r}}, \quad\|x\|_{p}=\left(\sum_{k=1}^{n}\left|x_{k}\right|^{p}\right)^{1 / p}, \quad 0 \leqslant p<\infty, \\
& \|x\|_{\infty}=\sup \left\{\left|x_{k}\right|: k=1, \cdots, n\right\} \quad \text { if } x=\operatorname{col}\left(x_{1}, \cdots, x_{n}\right) .
\end{aligned}
$$

In particular, Friedland [3], in generalizing the work of Nehari and Schwarz, shows that if $1<p<r \leqslant \infty$ then

$$
c_{r p}(n)=2 \int_{0}^{1}\left(1+\tau^{r}\right)^{-(1 / r)}\left(1+\tau^{q}\right)^{-(1 / q)} d \tau, \quad p^{-1}+q^{-1}=1 ;
$$

also

$$
\begin{aligned}
c_{r^{\infty}}(2 m) & =2 m^{-(1 / r)} \int_{0}^{1}\left[\left(1+\tau^{r}\right)^{(1 / r)}(1+\tau)\right]^{-1} d \tau, \\
c_{r^{\infty}}(2 m+1) & =m^{-(1 / r)} \int_{0}^{1}\left[\left(\tau^{r}+\frac{m+1}{m}\right)^{-(1 / r)}\right. \\
& \left.+\left(\frac{m+1}{m} \tau^{r}+1\right)^{-(1 / r)}\right](1+\tau)^{-1} d \tau .
\end{aligned}
$$

These results are also announced in [2]. In this note a special case of (9) is used, namely, when $A=y z^{T}$ where

$$
y(t)=\operatorname{col}\left(y_{1}(t), \cdots, y_{n}(t)\right), \quad z(t)=\operatorname{col}\left(z_{1}(t), \cdots, z_{n}(t)\right) .
$$

Then (9) has the form

$$
\int_{a}^{b}\|y\|_{p}\|z\|_{s}<c_{r p}(n), \quad r^{-1}+s^{-1}=1 .
$$

Friedland's proof of the optimality of $c_{r p}(n)$ in the general case of (2) shows it is optimal in this case also. The lemma and (10) yield the following theorem.

THEOREM. Suppose that $u(t)=\operatorname{col}\left(u_{1}(t), \cdots, u_{n}(t)\right)$ is any system of functions satisfying (3) and $v(t)=\operatorname{col}\left(v_{1}(t), \cdots, v_{n}(t)\right)$ is given by (4). Then, if each of the operators $U_{k}$ defined by (5) is disconjugate on $[a, b]$,

$$
\int_{a}^{b}\|v\|_{p}\|L u\|_{s}<c_{r p}(n), \quad r^{-1}+s^{-1}=1
$$

implies $L$ is disconjugate on $[a, b]$.

This theorem may be interpreted as giving an estimate on the permissible size of a perturbation of a disconjugate operator

$$
W\left(u_{1}, \cdots, u_{n}, f\right) / W\left(u_{1}, \cdots, u_{n}\right)
$$

while preserving disconjugacy. Theoretically such estimates may be obtained by finding bounds for Green's functions of the operator being perturbed but these bounds are often difficult to obtain in practice. Many disconjugacy 
criteria in the literature pertain to perturbations of $D^{n}$ (i.e. the coefficients $p_{i}$ are required to be small in some sense) and usually give better results than (11) in that case if $\left(u_{1}, \cdots, u_{n}\right)$ is chosen from the null set of $D^{n}$ (for example, see Levin [5], Hartman [4], Willett [10], Fink [1]). However since the restrictions on $u$ are slight the present result offers a wide range of tests; for example with the choice $u(t)=\left(e^{\lambda_{1} t}, \cdots, e^{\lambda_{n} t}\right), \lambda_{i} \neq \lambda_{j}$ if $i \neq j, L$ is considered as a perturbation of $\left(D-\lambda_{1}\right) \ldots\left(D-\lambda_{n}\right)$ and with $p=s=2$ the following criterion is established.

CoRollary. If $L\left(e^{\lambda t}\right)=P(\lambda, t) e^{\lambda t},\left(\lambda_{1}, \cdots, \lambda_{n}\right)$ is any set of $n$ distinct real numbers and $\mu_{k}=\prod_{i \neq k}\left(\lambda_{i}-\lambda_{k}\right)^{-1}$, then

$$
\int_{a}^{b}\left[\left(\sum_{k=1}^{n} \mu_{k}^{2} e^{-2 \lambda_{k} t}\right)\left(\sum_{k=1}^{n} P\left(\lambda_{k}, t\right)^{2} e^{2 \lambda_{k} t}\right)\right]^{1 / 2} d t<\frac{\pi}{2}
$$

implies $L$ is disconjugate on $[a, b]$.

Since nonoscillation of (6) is not equivalent to disconjugacy of $L$ it is not clear if the numbers $c_{r p}(n)$ in (11) are best possible.

\section{REFERENCES}

1. A. M. Fink, Differential inequalities and disconjugacy, J. Math. Anal. Appl. 49 (1975), 758-772. MR 52 \#862.

2. S. Friedland, Nonoscillation and integral inequalities, Bull. Amer. Math. Soc. 80 (1974), 715-717. MR 49 \# 10963.

3. Nonoscillation, disconjugacy and integral inequalities, Mem. Amer. Math. Soc. No. 176 (1976), 1-78.

4. P. Hartman, On disconjugacy criteria, Proc. Amer. Math. Soc. 24 (1970), 374-381. MR 40 \#4535.

5. A. Ju. Levin, $A$ bound for a function with monotonely distributed zeros of successive derivatives, Mat. Sb. 64 (106) (1964), 396-409. (Russian) MR 29 \#4941.

6. Z. Nehari, Oscillation theorems for systems of linear differential equations, Trans. Amer. Math. Soc. 139 (1969), 339-347. MR 39 \# 542.

7. Nonoscillation and disconjugacy of systems of linear differential equations, J. Math. Anal. Appl. 42 (1973), 237-254. MR 47 \#8976.

8. G. Pólya, On the mean-value theorem corresponding to a given linear homogeneous differential equation, Trans. Amer. Math. Soc. 24 (1924), 312-324.

9. B. Schwarz, Norm conditions for disconjugacy of complex differential systems, J. Math. Anal. Appl 28 (1969), 553-568. MR 40 \#2963.

10. D. Willett, Generalized De la Vallée Poussin disconjugacy tests for linear differential equations, Canad. Math. Bull. 14 (1971), 419-428. MR 50 \#687.

Department of Mathematics, University of Alberta, Edmonton, Alberta T6G 2G1, CANADA 